\title{
Snail mediates invasion through uPA/uPAR and the MAPK signaling pathway in prostate cancer cells
}

\author{
DIANDRA D. RANDLE, SHINEKA CLARKE, VERONICA HENDERSON and VALERIE A. ODERO-MARAH \\ Department of Biological Sciences, Center for Cancer Research and Therapeutic Development, \\ Clark Atlanta University, Atlanta, GA 30314, USA
}

Received March 13, 2013; Accepted September 17, 2013

DOI: $10.3892 / \mathrm{ol} .2013 .1635$

\begin{abstract}
Epithelial-mesenchymal transition (EMT) is a process by which cancer cells acquire mesenchymal properties, such as induction of vimentin, while epithelial-associated genes like E-cadherin are lost. This enables cells to be more metastatic. Factors that are able to induce EMT include growth factors such as transforming growth factor- $\beta$ (TGF- $\beta$ ) and epidermal growth factor, and transcription factors such as Snail. Snail-induced EMT promotes migration and invasion and we hypothesized that this may be mediated by the activity of urokinase-type plasminogen activator (uPA) and its receptor (uPAR). LNCaP, 22Rv1 and ARCaP human prostate cancer (CaP) cells stably transfected with empty vector control (Neo) or constitutively active Snail exhibited increased cell invasion. Superarray analysis revealed an upregulation in UPA and UPAR RNA expression in Snail-transfected ARCaP cells compared with that of a Neo control. In addition, the protein expression levels of Snail, uPA and UPAR were measured by western blot analysis which showed that overexpression of Snail increased UPA and UPAR protein levels. The activity of UPA in conditioned media was measured using an ELISA which revealed that uPA activity was elevated in LNCaP, 22Rv1 and ARCaP cells overexpressing Snail. Additionally, transient silencing of UPAR in ARCaP cells overexpressing Snail using short interfering RNA resulted in abrogation of Snail-mediated invasion. Snail overexpression was associated with increased extracellular-signal-regulated kinase activity, and antagonism of this activity with mitogen-activated protein (MAPK)
\end{abstract}

Correspondence to: Dr Valerie A. Odero-Marah, Department of Biological Sciences, Center for Cancer Research and Therapeutic Development, Clark Atlanta University, 223 James P. Brawley Drive SW, Atlanta, GA 30314, USA

E-mail: vodero_marah@cau.edu

Abbreviations: EMT, epithelial to mesenchymal transition; uPA, urokinase plasminogen activator; uPAR, urokinase plasminogen activator receptor; $\mathrm{CaP}$, prostate cancer; MAPK, mitogen-activated protein kinase

Key words: Snail, invasion, urokinase plasminogen activator, urokinase plasminogen activator receptor, mitogen-activated protein kinase, prostate cancer inhibitor, UO126, inhibited cell invasion and decreased uPA activity. Therefore, Snail-mediated cell invasion in human CaP cells may occur via the regulation of $\mathrm{UPA} / \mathrm{uPAR}$ and the MAPK signaling pathway.

\section{Introduction}

Prostate cancer $(\mathrm{CaP})$ is the most commonly diagnosed malignancy in the United States, with the majority of cases occurring in males over the age of 55 (1). In 2012, 241,740 new cases of $\mathrm{CaP}$ were predicted to be diagnosed, with $\sim 28,170$ men succumbing to $\mathrm{CaP}$, in the United States alone (1). Tumors that are detected early via testing serum prostate-specific antigen levels or digital rectal examination may be effectively treated by prostatectomy or radiation therapy (2). Approximately $30 \%$ of treated patients suffer relapse and progress to hormone refractory prostate cancer (HRPC), which no longer responds to androgen ablation, whereas early $\mathrm{CaP}$ growth is androgen-dependent. At that stage, there is no curative therapy available for metastatic $\mathrm{CaP}(3,4)$. Metastasis is a complex process by which cancer cells leave the primary tumor and migrate to a secondary site where they recolonize. It consists of multiple steps that are interconnected, including invasion, migration, intravasation, extravasation and recolonization $(5,6)$. The shortcomings of treatment for such highly invasive and metastatic disease have led to several investigations of various molecular targets that directly affect invasion and metastasis with the aim of developing safe and effective treatments.

Numerous studies suggest that epithelial-mesenchymal transition (EMT) may be an important step leading to cancer metastasis (7-9). A notable mechanism by which E-cadherin is downregulated in EMT is transcriptional repression by Snail $(10,11)$. Induction of Snail expression has been noted in a number EMT processes that have been studied (11-13). Additionally, increases in signaling in survival pathways such as mitogen-activated protein kinase (MAPK) is associated with increased Snail expression (14). Snail is composed of two interacting domains $(12,15,16)$; the C-terminal domain is responsible for binding to DNA sequences with a 5'-CAGGTG-3' core, while the N-terminal is required for transcriptional repression $(16,17)$. Overexpression of Snail is sufficient to induce EMT and is associated with highly invasive tumors in mice and humans (18). 
In order for tumors to colonize to a secondary site, they must invade the extracellular matrix (ECM) $(5,6)$. Several proteolytic enzymes are involved in this process of degradation. Among these enzymes is the plasminogen activation (PA) system which leads to activation of matrix metalloproteases (MMPs) $(19,20)$. The members of the PA system include urokinase-type plasminogen activator (uPA), plasminogen activator inhibitors (PAIs) and the uPA receptor (uPAR) $(19,20)$. uPA, when bound to its cellular receptor uPAR, efficiently converts plasminogen into the broad-spectrum serine protease plasmin; its action on plasminogen is controlled by the serine protease inhibitors PAI-1 and PAI-2 (13-15). uPA catalyzes the activation of plasminogen into plasmin by cleaving the arginine-valine bond. In turn, plasmin facilitates the release of several proteolytic enzymes, including gelatinase and fibronectin (19-21).

It has been well established that UPA and UPAR, both members of the PA system, are involved in cancer invasion and metastases (19-23). It has been shown that plasma levels of UPA and UPAR are higher in males with CaP compared with healthy controls and significantly declined after prostate removal (24). Under normal conditions, uPAR is considered to have fairly limited tissue expression (25). Studies using mice and human clinical samples have identified conditions in which UPAR expression is induced $(25,26)$. uPAR is induced during ECM remodeling, stress, injury and inflammation, and is highly expressed during tissue reorganization and inflammation, as well as in virtually all human cancers $(19,21,25)$. Furthermore, it has been shown that uPAR is under an extracellular-signal-regulated kinase (ERK)-dependent mechanism and blocking uPAR's activity leads to inhibition of motility in hepatocellular carcinoma (27). In human gastric cancer, studies have demonstrated that epidermal growth factor (EGF) stimulates uPAR expression via the ERK pathway, sequentially increasing cell invasion (28).

Several studies have shown that Snail mediates invasion through MMP activation (29-31); however, there are few studies that link Snail and uPA to cancer progression. One study indicated that silencing UPA expression in MDA-MB-231 breast cancer cells decreased expression of vimentin and Snail, and induced changes in morphology characteristic of epithelial cells (32). These results demonstrate that uPAR-initiated cell signaling may be targeted to reverse EMT in cancer (32). Another study suggested that when Snail is blocked in the invasive breast cancer cell-line MDA-MB-231, there is a decrease in the expression of PAI- 1 and uPA transcripts and reduced migration (33).

Previously, we have stably overexpressed Snail in LNCaP and ARCaP CaP cell lines and shown that Snail led to EMT associated with decreased/relocalized E-cadherin, increased vimentin and increased migration (34-37). In this study, we investigated the molecular mechanisms of Snail-mediated cell invasion. We propose that Snail increases invasion via uPA/uPAR signaling. The results showed that Snail overexpression led to an increase in cell invasion, which was antagonized by uPAR silencing. Snail also increased the levels of UPA and UPAR protein, as well as UPA and ERK activities. Furthermore, the inhibition of MAPK activity decreased UPA activity and cell invasion. Our results show, for the first time, a link between Snail, MAPK and UPA/uPAR in CaP. This demonstrates that Snail regulates cell invasion via uPA-uPAR activites, possibly through the MAPK pathway.

\section{Materials and methods}

Reagents and antibodies. RPMI-1640 medium and penicillin/streptomycin were purchased from VWR International, Inc. (West Chester, PA, USA). The protease inhibitor cocktail was obtained from Roche Molecular Biochemicals (Indianapolis, IN, USA), while G418 and anti-human actin antibodies were purchased from Sigma-Aldrich, Inc. (St. Louis, MO, USA), and rabbit polyclonal anti-human Snail antibody and rabbit anti-phospho-ERK1/2 (p-ERK) were obtained from Cell Signaling Technology, Inc. (Danvers, MA, USA). Rabbit polyclonal anti-uPA, anti-uPAR and anti-total-ERK1/2 were purchased from Santa Cruz Biotechnology, Inc. (Santa Cruz, CA, USA). Horseradish peroxidase-conjugated sheep anti-mouse, sheep anti-rabbit and the ECL Prime or ECL Plus chemiluminescent reagents were obtained from GE Healthcare Life Sciences (Little Chalfont, UK. Fetal bovine serum (FBS) and dextran-coated charcoal-treated FBS (DCC-FBS) were supplied by HyClone (South Logan, UT, USA). Control and Snail short interfering RNA (siRNA) constructs were purchased from Dharmacon, Inc. (Lafayette, CO, USA), and UO126 was purchased from Sigma-Aldrich, Inc. The uPA Activity Assay kit was obtained from Millipore (Billerica, MA, USA) and Matrigel was purchased from BD Biosciences (Bedford, MA, USA).

Cell culture. Human CaP cell line ARCaP (Cedar Sinai Medical Center, Los Angeles, CA, USA) stably transfected with constitutively active Snail cDNA (ARCaP Snail representing an aggressive cell line) or an empty vector $\mathrm{Neo}$ (ARCaP Neo representing the less aggressive cell line), as well as LNCaP cells overexpressing Snail, have been previously described as representing an EMT model and were utilized in these experiments (34-37). The 22Rv1 cells overexpressing Snail utilized in the present experiments were previously generated (35). The $\mathrm{LNCaP}$ human $\mathrm{CaP}$ cell line was obtained from American Type Culture Collection (Manassas, VA, USA) and maintained in RPMI-1640 (Corning Cellgro, Manassas, VA, USA), supplemented with $10 \%$ FBS, $1 \%$ non-essential amino acids and $1 \%$ antibiotics at $37^{\circ} \mathrm{C}$ in $5 \% \mathrm{CO}_{2}$. The Snail-transfected cells were maintained in RPMI-1640 supplemented with $10 \%$ FBS, $1 \%$ non-essential amino acids and $1 \%$ antibiotics plus $400 \mu \mathrm{g} / \mathrm{ml} \mathrm{G} 418$. All cells were maintained at $70-80 \%$ confluence.

Western blot analysis. Cells were cultured to $85-90 \%$ confluency; subsequently, cells were washed with phosphate-buffered saline and harvested in modified RIPA buffer $(50 \mathrm{mM}$ Tris, $\mathrm{pH} 8.0 ; 150 \mathrm{mM} \mathrm{NaCl} ; 0.02 \% \mathrm{NaN}_{3} ; 0.1 \%$ sodium dodecyl sulfate; $1 \%$ NP-40; $0.5 \%$ sodium deoxycholate) containing $1.5 \mathrm{X}$ protease inhibitor cocktail, $1 \mathrm{mM}$ phenylmethylsufonyl fluoride and $1 \mathrm{mM}$ sodium orthovanadate. Protein concentrations were calculated using the bicinchoninic acid protein assay (Pierce, Rockford, IL, USA). Equal concentrations of whole cell protein were separated on a $10 \%$ SDS-polyacrylamide gel electrophoresis gel and transferred to a nitrocellulose membrane. Non-specific antibody binding sites were blocked 
using 3 or $5 \%$ non-fat dry milk and Tris-buffered saline and Tween-20 (TBST), and washed with TBST. Membranes were incubated with primary antibodies in $3 \%$ bovine serum albumin-TBST (p-ERK and Snail), or 5\% non-fat dry milk and TBST (uPA, uPAR, ERK1/2 and $\beta$-actin) overnight at $4^{\circ} \mathrm{C}$. Membranes were washed in TBST and incubated with HRP-conjugated sheep anti-rabbit (Snail, uPA, uPAR and p-ERK) or anti-mouse (actin) secondary antibody, then washed in TBST. Immunoblots were detected using ECL Prime or ECL Plus chemiluminescent reagent (GE Healthcare, Pittsburgh, PA, USA).

uPA activity assay. uPA activity was measured in conditioned medium from the human $\mathrm{CaP}$ cell sublines LNCaP Neo/Snail, ARCaP Neo/Snail and 22Rv1 Neo/Snail using the uPA activity assay kit according to the manufacturer's instructions. A chromogenic substrate is cleaved by active uPA to produce a colored product, which is detected on a plate reader at $405 \mathrm{~nm}$. The concentration of active uPA was calculated relative to standards provided with the kit.

siRNA transfection. Transient transfection of uPAR siRNA was performed on ARCaP Snail cells using DharmaFECT 1 reagent. Cells $\left(1 \times 10^{6} /\right.$ well) were seeded in a six-well plate and transfected with $200 \mathrm{~nm}$ uPAR-siRNA or control-siRNA in serum free media at $37^{\circ} \mathrm{C}$ with $5 \% \mathrm{CO}_{2}$ for $5 \mathrm{~h}$, followed by replacement of transfection media with RPMI-1640 supplemented with 5\% DCC-FBS. After $72 \mathrm{~h}$, transfected cells were harvested for western blot analysis of Snail, uPA, uPAR and $\beta$-actin; conditioned media was collected for the uPA activity assay. Transfected cells were also utilized for a subsequent invasion assay.

Invasion assay. The invasive properties of the cell lines were measured using the BD BioCoat ${ }^{\mathrm{TM}}$ Matrigel $^{\mathrm{TM}}$ Invasion guidelines. Briefly, Boyden chamber inserts (Thermo Fisher Scientific, Waltham, MA, USA) were coated with $50 \mu 1$ 1:4 Matrigel and allowed to solidify at $37^{\circ} \mathrm{C}$ for $1 \mathrm{~h}$. Cells were seeded in quadruplicate at $5 \times 10^{4}$ (for ARCaP and 22Rv1) and $1 \times 10^{5}$ (for $\mathrm{LNCaP}$ ) in $0.1 \% \mathrm{FBS}$, while the lower chamber contained $10 \%$ FBS. Cells were treated accordingly and allowed to invade through the porous membrane coated with Matrigel at $37^{\circ} \mathrm{C}$ for $24-72 \mathrm{~h}$. Inserts were fixed, stained and photographed in two fields per insert. Cell counts were performed for the determination of relative invasion or the stain solubilized with Sorenson solution and optical density measured at $590 \mathrm{~nm}$.

ERK inhibitor asssay treatments. The human CaP cell subline ARCaP Snail $\left(1 \times 10^{6}\right)$, was cultured overnight. The following day, cells were treated with $20 \mu \mathrm{M}$ ERK1/2 inhibitor (U0126) at the following time-points (0 and $30 \mathrm{~min}, 2$, 6, 24 and $72 \mathrm{~h}$ ). The conditioned media was collected and whole cell lysates were collected as previously described.

Superarray analysis. Total RNA was isolated from ARCaP Neo or ARCaP Snail cells using the Qiagen kit according to the manufacturer's instructions and $1 \mu \mathrm{g}$ of which was reverse transcribed with oligo(dT) using MMLV-reverse transcriptase (Invitrogen Life Technologies, Carlsbad, CA, USA), to generate cDNA. The labeled cDNA was incubated with GEArray Q Series cancer pathway membranes (SuperArray, Valencia, CA, USA) at $60^{\circ} \mathrm{C}$ overnight. The membrane used in the present study contained 96 genes that were closely associated with cancer pathways, in addition to housekeeping control genes (such as GAPDH). After being washed, the membrane was incubated with streptavidin-alkaline phosphatase and was finally exposed to CDP-Star chemiluminescent substrate (SuperArray). Signal detection was performed using a high Performance chemiluminescence film (Amersham Biosciences, Amersham, UK). Analysis of results was performed using GEArray Expression Analysis Suite software (http://geasuite.superarray.com).

Statistical analysis. All data are presented as the mean \pm standard error of at least three independent experiments. The data were analyzed using two-way analysis of variance or Student's t-test. All statistical analyses were performed and all graphs generated using GraphPad Prism 5.0 software (GraphPad Software Inc., San Diego, CA, USA). P $<0.05$ was considered to indicate statistically significant differences.

\section{Results}

Overexpression of Snail leads to an increase in cell invasion. Previously, we have shown that Snail overexpression increases cell invasion in 22Rv1 cells (35). To confirm these results and examine the effect of Snail overexpression on LNCaP and ARCaP invasion through the ECM, an invasion assay was performed where Matrigel mimicked the ECM. As expected, Snail-transfected cells exhibited significantly more cell invasion compared with the Neo control-transfected cells in all three cell lines tested (Fig. 1). Therefore, Snail is associated with increased cell invasion.

Overexpression of Snail leads to an upregulation of $u P A$ and $U P A R$. In order to examine the molecular mechanism by which Snail may increase cell invasion, a superarray analysis was performed on ARCaP Neo and ARCaP Snail CaP cells to identify genes downstream of Snail that may be responsible for the increase in cell invasion. Notably, a protein associated with cell invasion, uPA, and its receptor, uPAR, were upregulated (Fig. 2A). Subsequently, the protein expression levels of uPAR and its ligand uPA were evaluated in Snail overexpressing LNCaP, 22Rv1 and ARCaP cells. In all three CaP lines, Snail transfection increased UPA and UPAR protein expression (Fig. 2B). Additionally, measurement of secreted uPA activity in conditioned media showed that LNCaP, 22Rv1 and ARCaP cell lines overexpressing Snail exhibited higher uPA activity compared with that of the Neo control (Fig. 2C). The results also suggested that the androgen-independent ARCaP cells had a higher active uPA concentration compared with that of the androgen-dependent LNCaP and 22Rv1 cells. Therefore, Snail is associated with increased $\mathrm{uPa} / \mathrm{uPAR}$ protein levels and increased uPA activity.

uPAR knockdown in Snail-overexpressing ARCaP cells leads to decreased cell invasion. To evaluate the contribution of uPA/uPAR in the increased invasion that was observed in the Snail overexpressed cells, uPAR was transiently knocked 
A

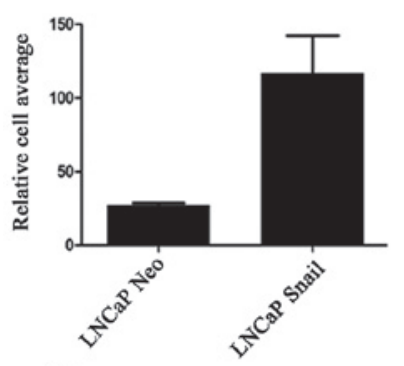

B

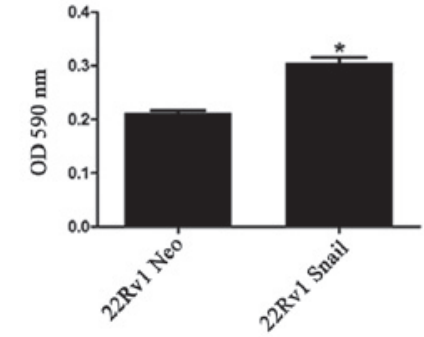

C

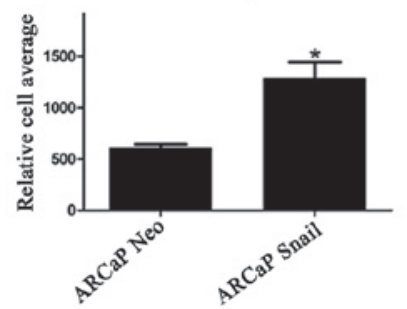

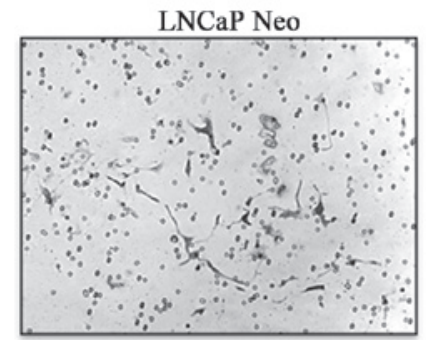

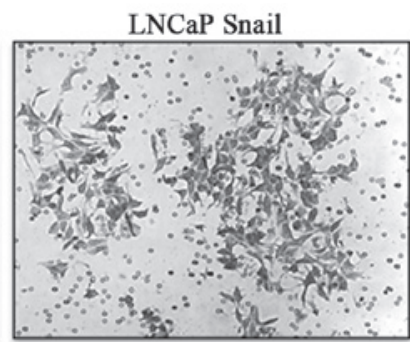

ARCaP Neo

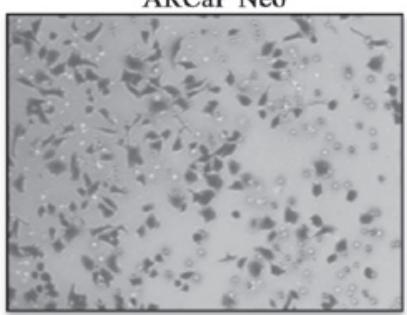

\section{ARCaP Snail}

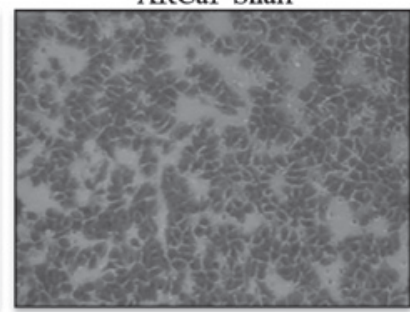

Figure 1. Snail overexpression increases cell invasion in prostate cancer cells. Prostate cancer cells lines, LNCaP, $22 \mathrm{RV} 1$ and ARCaP, stably transfected with empty vector (Neo) or constitutively active Snail cDNA using Lipofectamine 2000 were utilized for cell invasion assays using the Boyden chamber. (A) LNCaP Neo and Snail. Magnification, x10. (B) 22RV1 Neo and Snail and (C) ARCaP Neo and Snail were plated at a density of 5x104 cells in culture inserts coated with Matrigel. Magnification, x10. Cells that invaded were stained with crystal violet and either counted or solubilized with Sorenson solution, and optical density (OD) was measured at $590 \mathrm{~nm}$. These studies were performed in triplicate and the average number of cells from each repeat that invaded the Matrigel were deemed the 'relative cell average.' Results are representative of three independent experiments. Statistical analysis was performed using two-way analysis of variance or Student's t-test ('P $<0.05)$.

A

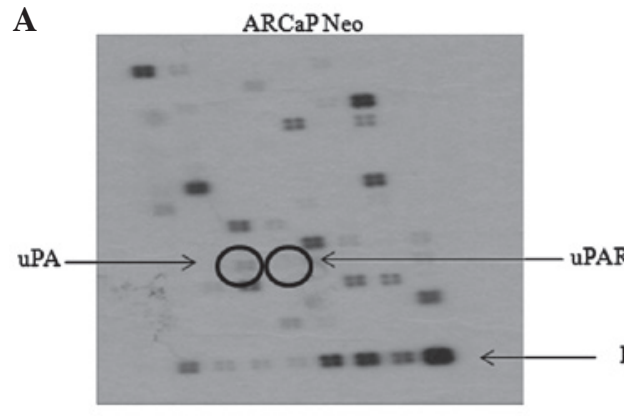

B

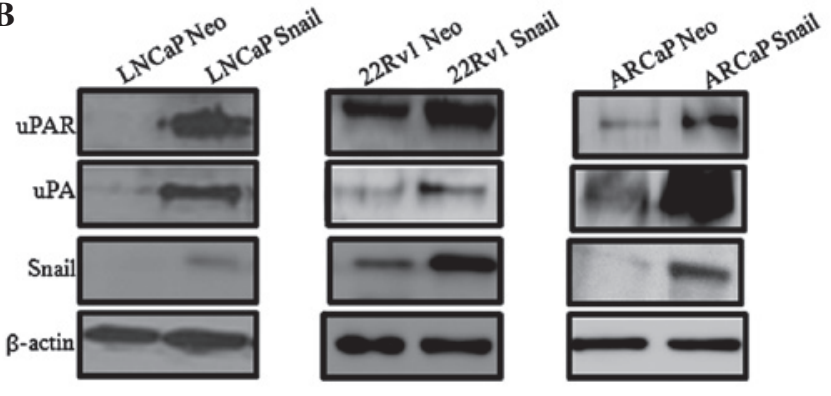

ARCaP Snail

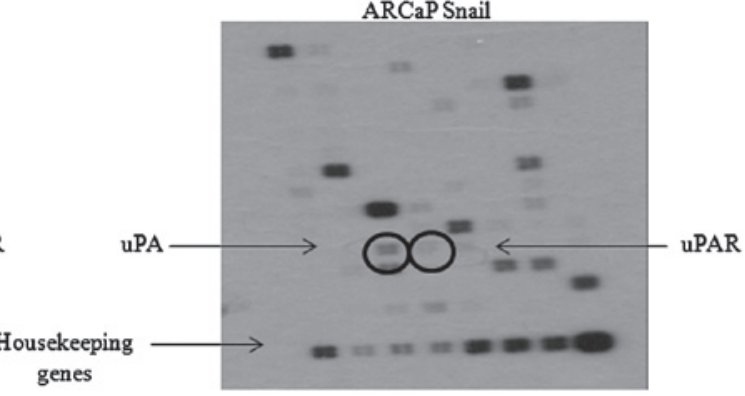

C

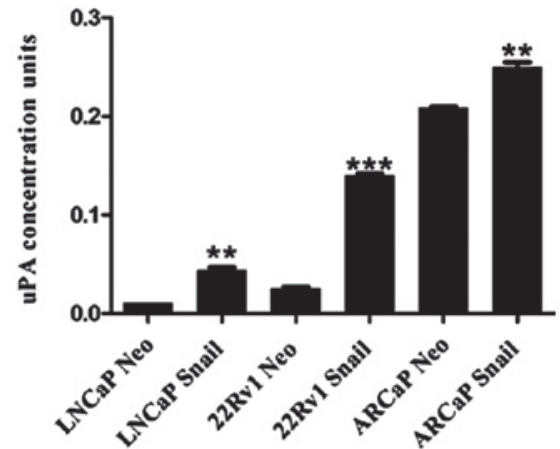

Figure 2. Snail increases urokinase-type plasminogen activator (uPA)/uPA receptor (uPAR) levels and uPA activity in prostate cancer cells. (A) Total RNA was isolated from ARCaP empty vector (Neo) or ARCaP Snail cells. Superarray analysis was performed utilizing gene expression arrays for cancer pathways. uPA and uPAR RNA levels were upregulated by Snail. (B) Snail, uPA and UPAR protein expression was determined by western blot analysis. Actin was utilized as the loading control. (C) Secreted uPA activity was measured in conditioned media using uPA activity assay. Results are representative of three independent experiments. Statistical analysis was performed using two-way analysis of variance or Student's t-test $\left({ }^{* *} \mathrm{P}<0.01\right.$ and $\left.{ }^{* * *} \mathrm{P}<0.001\right)$. 
A

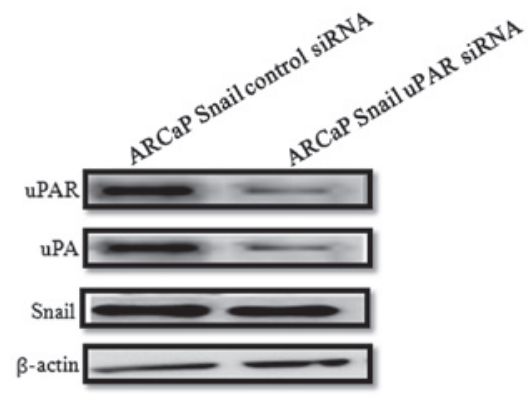

B
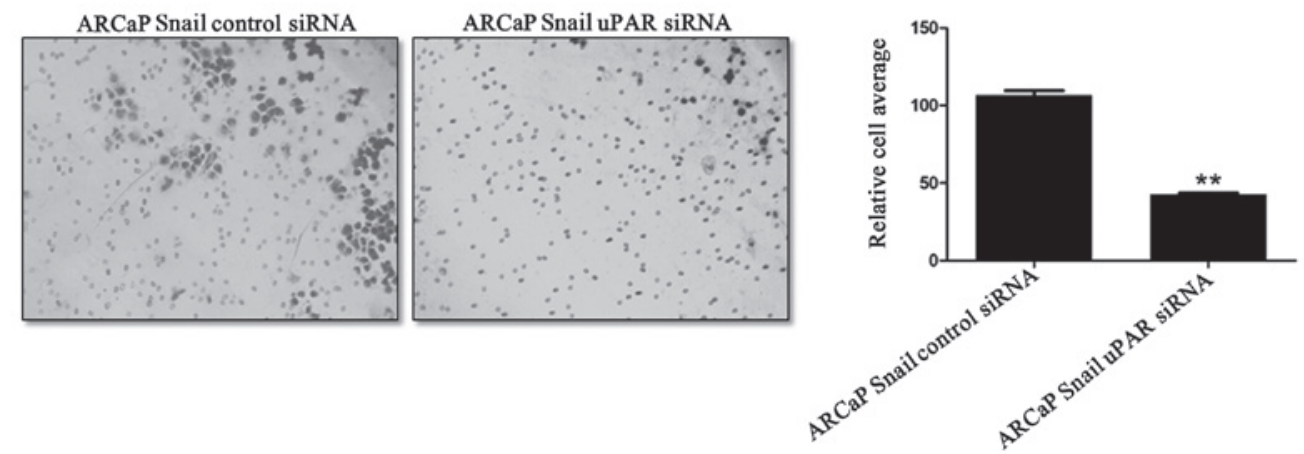

Figure 3. Snail mediates cell invasion via urokinase-type plasminogen activator receptor (uPAR). Transient transfection of control or uPAR short interfering RNA (siRNA) was performed on ARCaP Snail cells using DharmaFECT 1 reagent. (A) uPA, uPAR and Snail expression was determined by western blot analysis. Actin was utilized as the loading control. (B) Invasion through Matrigel was tested on the cells treated with control siRNA and uPAR siRNA using the Boyden chamber. Results are representative of three independent experiments. Cells were stained with crystal violet and rinsed in distilled $\mathrm{H}_{2} \mathrm{O}$. Magnification, x10. These studies were performed in triplicate and the average number of cells from each repeat that invaded the Matrigel were deemed the 'relative cell average.' Statistical analysis was performed using two-way analysis of variance or Student's t-test $\left({ }^{* *} \mathrm{P}<0.01\right)$.

A

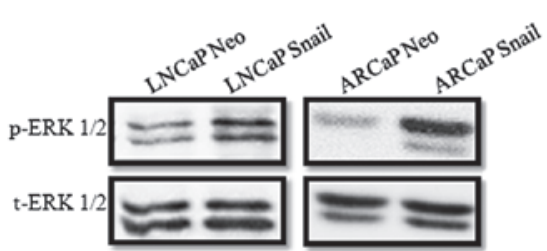

$\mathbf{C}$

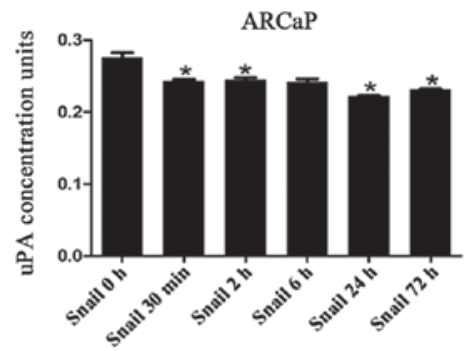

D

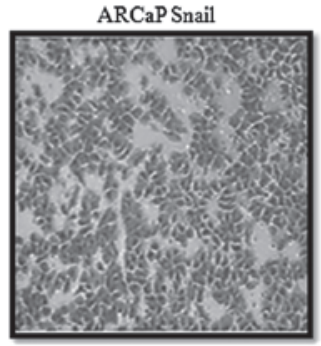

B

ARCaP Snail

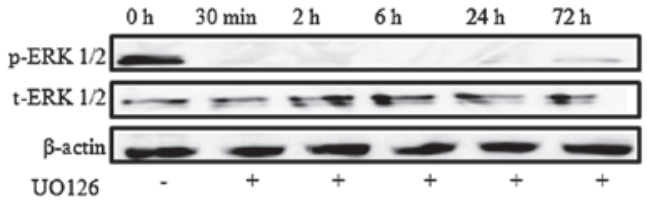

U0126
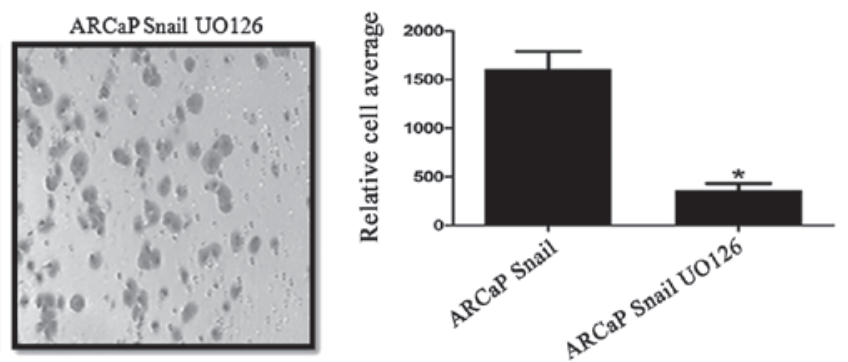

Figure 4. Extracellular-signal-regulated kinase (ERK) inhibition antagonizes Snail-mediated urokinase-type plasminogen activator (uPA) activity and cell invasion. (A) Phospho-ERK1/2 (p-ERK), total ERK1/2 (t-ERK) and Snail expression were determined by western blot analysis in LNCaP and ARCaP cells overexpressing Snail. Actin was utilized as the loading control. (B) ARCaP Snail cells were treated with UO126 MEK inhibitor for various time periods. (C) Secreted active uPA activity was measured in conditioned media using uPA activity assay. (D) Invasion through Matrigel was tested on ARCaP Snail cells without and with UO126 for $24 \mathrm{~h}$ using the Boyden chamber. Cells were stained with crystal violet and rinsed in distilled $\mathrm{H}_{2} \mathrm{O}$. Magnification, $\mathrm{x} 10$. These studies were performed in triplicate and the average number of cells from each repeat that invaded the Matrigel were deemed the 'relative cell average.' Results are representative of three independent experiments. Statistical analysis was performed using two-way analysis of variance or Student's t-test ("P<0.05). 
down in ARCaP Snail cells. Western blot analysis confirmed the knockdown of uPAR (Fig. 3A). Of note, uPAR knockdown was accompanied by a decrease in uPA expression, while Snail expression was not affected by this knockdown (Fig. 3A). Functionally, there was a significant decrease in invasion following uPAR knockdown (Fig. 3B). Thus, uPAR contributes to Snail-mediated cell invasion.

Inhibition of MAPK activity downregulates $u P A$ activity and decreases cell invasion. We have previously demonstrated that there is an increase of phosphorylated MAPK (p-ERK) in CaP cells overexpressing Snail $(34,36)$. Therefore, we investigated whether Snail regulation of uPA activity was mediated by MAPK signaling. It was identified that Snail overexpression increases ERK activity in LNCaP and ARCaP cell lines (Fig. 4A). Subsequently, Snail-transfected ARCaP cells were treated with $20 \mu \mathrm{M}$ UO126 MEK inhibitor for $30 \mathrm{~min}$ and 2, 6, 24 and $72 \mathrm{~h}$. Decreased ERK activity was observed by $30 \mathrm{~min}$ and persisted until $72 \mathrm{~h}$ as shown by the western blot analysis (Fig. 4B). It was also revealed that inhibiting MAPK activity significantly decreased uPA activity within $30 \mathrm{~min}$ (Fig. 4C). Finally, ARCaP Snail cells treated with U1O26 for $24 \mathrm{~h}$ showed decreased invasive potential compared with that of the ARCaP Neo control (Fig. 4D).

\section{Discussion}

Studies have suggested that epithelial mesenchymal transition (EMT) is an important step leading to cancer metastasis (7-9). One mechanism by which E-cadherin is downregulated in EMT is transcriptional repression by Snail $(10,11)$. In the present study, we have shown that overexpression of Snail increases cell invasion in androgen-dependent LNCaP and 22RV1 prostate cancer cell lines and androgen-independent ARCaP prostate cancer cell lines. In Snail-transfected ARCaP cells, certain genes that were upregulated and downregulated were evaluated via superarray analysis, based on their function. The results of the superarray demonstrated that the overexpression of Snail leads to upregulation of genes involved with invasion and metastasis, such as uPA and UPAR. It was noteworthy that UPA and UPAR were upregulated in Snail-transfected CaP cells, as in previous studies performed in PC3 and DU145 cells, RNA interference of UPA and UPAR resulted in UPA and UPAR mRNA and protein expression being completely inhibited and there was a decline in metastasis (38). Although the signaling cascade resulting in the expression of UPA and UPAR being downregulated was not determined, the superarray analysis and UPAR siRNA studies done in Snail-transfected cells suggest that it may be through Snail. To confirm our superarray studies, we showed that UPA and UPAR protein expression was increased in Snail-overexpressing cells. Additionally, Snail overexpression led to increased uPA activity. Although there was a general increase in uPA activity in the Snail-transfected cells, there was a greater level of uPA activity in the androgen-independent ARCaP cells compared with that in the androgen-dependent LNCaP and 22Rv1 cells.

To determine the effect the UPA/UPAR system has on the increase in invasion in Snail-transfected cells, uPAR was transiently knocked down. The most well known activator of uPA is UPAR; therefore; knocking down UPAR inhibits the function of both uPA and uPAR (19). We observed that UPAR knockdown in ARCaP Snail cells led to a significant decrease in cell invasion. It is noteworthy that Snail expression was not affected by the knockdown of UPAR, suggesting that UPAR is acting downstream of Snail to increase cell invasion; thus, for the first time, we show that Snail relies on UPAR to increase invasion. It may be suggested that UPA/uPAR signaling alone does not have an important role in Snail-mediated invasion in ARCaP cells, as uPAR knockdown did not completely eliminate invasion. Previously, we have shown that ERK activity is increased in Snail-transfected ARCaP cells $(34,36)$. In the present study, in order to determine whether Snail mediates invasion through the MAPK pathway, Snail-transfected cells were treated with MEK inhibitor UO126 for various time periods. uPA activity and invasion was significantly decreased in ARCaP Snail cells treated with UO126 in a time-dependent manner. This suggests that Snail may use the MAPK pathway to mediate cell invasion through uPA/uPAR signaling in ARCaP cells. Supporting these results, the literature suggest that UPAR is under an ERK-dependent mechanism and blocking UPAR's activity leads to inhibition of motility in hepatocellular carcinoma (27). Additionally, a study on human gastric cancer has shown that EGF stimulates uPAR expression via the ERK pathway, sequentially increasing cell invasion (28). Although the activity of uPA was decreased upon MAPK inhibition, it was not completely eliminated, possibly since its activity may be mediated by additional pathways, such as AKT. In breast cancer, studies have shown that upon uPA binding to UPAR, AKT is activated $(39,40)$.

Overall, the present results show, for the first time, a link between Snail, MAPK and UPA/uPAR in CaP. Our studies suggest that Snail overexpression increases cell invasion through the upregulation of UPA/uPAR signaling, which is mediated in part by the MAPK signaling pathway.

\section{Acknowledgements}

This study was supported by NIH grants 1P20MD002285 (VOM) and 8G12MD007590.

\section{References}

1. Siegel R, Naishadham D and Jemal A: Cancer statistics, 2012. CA Cancer J Clin 62: 10-29, 2012.

2. Siegel R, DeSantis C, Virgo K, et al: Cancer treatment and survivorship statistics, 2012. CA Cancer J Clin 62: 220-241, 2012.

3. Mimeault $M$ and Batra SK: Recent advances on multiple tumorigenic cascades involved in prostatic cancer progression and targeting therapies. Carcinogenesis 27: 1-22, 2006.

4. Culig Z, Steiner H, Bartsch G and Hobisch A: Mechanisms of endocrine therapy-responsive and -unresponsive prostate tumours. Endocr Relat Cancer 12: 229-244, 2005.

5. Clarke NW, Hart CA and Brown MD: Molecular mechanisms of metastasis in prostate cancer. Asian J Androl 11: 57-67, 2009.

6. Chambers AF, MacDonald IC, Schmidt EE, et al: Steps in tumor metastasis: new concepts from intravital videomicroscopy. Cancer Metastasis Rev 14: 279-301, 1995.

7. Xu J, Lamouille S and Derynck R: TGF-beta-induced epithelial to mesenchymal transition. Cell Res 19: 156-172, 2009.

8. Jing Y,Han Z,Zhang S, Liu Y and Wei L: Epithelial-Mesenchymal Transition in tumor microenvironment. Cell Biosci 1: 29, 2011.

9. Larue L and Bellacosa A: Epithelial-mesenchymal transition in development and cancer: role of phosphatidylinositol 3' kinase/AKT pathways. Oncogene 24: 7443-7454, 2005. 
10. Cho HJ, Baek KE, Saika S, Jeong MJ and Yoo J: Snail is required for transforming growth factor-beta-induced epithelial-mesenchymal transition by activating PI3 kinase/Akt signal pathway. Biochem Biophys Res Commun 353: 337-343, 2007.

11. Batlle E, Sancho E, Francí C, et al: The transcription factor snail is a repressor of E-cadherin gene expression in epithelial tumour cells. Nat Cell Biol 2: 84-89, 2000.

12. Nieto MA: The snail superfamily of zinc-finger transcription factors. Nat Rev Mol Cell Biol 3: 155-166, 2002.

13. Haraguchi M: The role of the transcriptional regulator snail in cell detachment, reattachment and migration. Cell Adh Migr 3 259-263, 2009

14. Barrallo-Gimeno A and Nieto MA: The Snail genes as inducers of cell movement and survival: implications in development and cancer. Development 132: 3151-3161, 2005

15. Domínguez D, Montserrat-Sentís B, Virgós-Soler A, et al: Phosphorylation regulates the subcellular location and activity of the snail transcriptional repressor. Mol Cell Biol 23: 5078-5089, 2003.

16. Peiró S, Escrivà M, Puig I, et al: Snaill transcriptional repressor binds to its own promoter and controls its expression. Nucleic Acids Res 34: 2077-2084, 2006.

17. Peinado H, Ballestar E, Esteller M and Cano A: Snail mediates E-cadherin repression by the recruitment of the Sin3A/histone deacetylase 1 (HDAC1)/HDAC2 complex. Mol Cell Biol 24 306-319, 2004.

18. Heebøll S, Borre M, Ottosen PD, Dyrskjøt L, Orntoft TF and Tørring N: Snaill is over-expressed in prostate cancer. APMIS 117: 196-204, 2009.

19. Dass K, Ahmad A, Azmi AS, Sarkar SH and Sarkar FH: Evolving role of uPA/uPAR system in human cancers. Cancer Treat Rev 34: 122-136, 2008

20. Conese $\mathrm{M}$ and Blasi F: Urokinase/urokinase receptor system: internalization/degradation of urokinase-serpin complexes: mechanism and regulation. Biol Chem Hoppe Seyler 376 143-155, 1995.

21. Smith HW and Marshall CJ: Regulation of cell signalling by uPAR. Nat Rev Mol Cell Biol 11: 23-36, 2010.

22. Dong Z, Saliganan AD, Meng H, et al: Prostate cancer cell-derived urokinase-type plasminogen activator contributes to intraosseous tumor growth and bone turnover. Neoplasia 10: 439-449, 2008.

23. Lee KH, Kim SW and Kim JR: Reactive oxygen species regulate urokinase plasminogen activator expression and cell invasion via mitogen-activated protein kinase pathways after treatment with hepatocyte growth factor in stomach cancer cells. J Exp Clin Cancer Res 28: 73, 2009.

24. Shariat SF, Roehrborn CG, McConnell JD, et al: Association of the circulating levels of the urokinase system of plasminogen activation with the presence of prostate cancer and invasion, progression, and metastasis. J Clin Oncol 25: 349-355, 2007.

25. Mazar AP, Ahn RW and O'Halloran TV: Development of novel therapeutics targeting the urokinase plasminogen activator receptor (uPAR) and their translation toward the clinic. Curr Pharm Des 17: 1970-1978, 2011.

26. Jo M, Takimoto S, Montel V and Gonias SL: The urokinase receptor promotes cancer metastasis independently of urokinase-type plasminogen activator in mice. Am J Pathol 175: 190-200, 2009
27. Bessard A, Frémin C, Ezan F, Coutant A and Baffet G: MEK/ERK-dependent uPAR expression is required for motility via phosphorylation of P70S6K in human hepatocarcinoma cells. J Cell Physiol 212: 526-536, 2007.

28. Baek MK, Kim MH, Jang HJ, et al: EGF stimulates uPAR expression and cell invasiveness through ERK, AP-1, and NF- $\mathrm{BB}$ signaling in human gastric carcinoma cells. Oncol Rep 20: $1569-1575,2008$

29. Jordà M, Olmeda D, Vinyals A, et al: Upregulation of MMP-9 in MDCK epithelial cell line in response to expression of the Snail transcription factor. J Cell Sci 118: 3371-3385, 2005.

30. Miyoshi A, Kitajima Y, Sumi K, et al: Snail and SIP1 increase cancer invasion by upregulating MMP family in hepatocellular carcinoma cells. Br J Cancer 90: 1265-1273, 2004.

31. Yokoyama K, Kamata N, Fujimoto R, et al: Increased invasion and matrix metalloproteinase-2 expression by Snail-induced mesenchymal transition in squamous cell carcinomas. Int $\mathrm{J}$ Oncol 22: 891-898, 2003.

32. Jo M, Lester RD, Montel V, Eastman B, Takimoto S and Gonias SL: Reversibility of epithelial-mesenchymal transition (EMT) induced in breast cancer cells by activation of urokinase receptor-dependent cell signaling. J Biol Chem 284: 22825-22833, 2009.

33. Fabre-Guillevin E, Malo M, Cartier-Michaud A, et al: PAI-1 and functional blockade of SNAI1 in breast cancer cell migration. Breast Cancer Res 10: R100, 2008.

34. Neal CL, McKeithen D and Odero-Marah VA: Snail negatively regulates cell adhesion to extracellular matrix and integrin expression via the MAPK pathway in prostate cancer cells. Cell Adh Migr 5: 249-257, 2011.

35. Neal CL, Henderson V, Smith BN, et al: Snail transcription factor negatively regulates maspin tumor suppressor in human prostate cancer cells. BMC Cancer 12: 336, 2012.

36. Barnett P, Arnold RS, Mezencev R, Chung LW, Zayzafoon M and Odero-Marah V: Snail-mediated regulation of reactive oxygen species in ARCaP human prostate cancer cells. Biochem Biophys Res Commun 404: 34-39, 2011.

37. McKeithen D, Graham T, Chung LW and Odero-Marah V: Snail transcription factor regulates neuroendocrine differentiation in LNCaP prostate cancer cells. Prostate 70: 982-992, 2010.

38. Pulukuri SM, Gondi CS, Lakka SS, et al: RNA interference-directed knockdown of urokinase plasminogen activator and urokinase plasminogen activator receptor inhibits prostate cancer cell invasion, survival, and tumorigenicity in vivo. J Biol Chem 280: 36529-36540, 2005

39. Alfano D, Iaccarino I and Stoppelli MP: Urokinase signaling through its receptor protects against anoikis by increasing BCL-xL expression levels. J Biol Chem 281: 17758-17767, 2006.

40. Lester RD, Jo M, Montel V, Takimoto S and Gonias SL: uPAR induces epithelial-mesenchymal transition in hypoxic breast cancer cells. J Cell Biol 178: 425-436, 2007. 